\title{
CRACK FRONT WAVES IN DYNAMIC FRACTURE
}

\author{
J. Fineberg ${ }^{1}$, E. Sharon ${ }^{2}$ and G. Cohen ${ }^{1}$ \\ ${ }^{1}$ The Racah Institute of Physics, The Hebrew University of Jerusalem, \\ Givat Ram, Jerusalem 91904, Israel \\ ${ }^{2}$ The Center for Nonlinear Dynamics, The University of Texas, Austin \\ 78712, TX, USA.
}

\begin{abstract}
A rapidly moving crack in a brittle material is often idealized as a one-dimensional object moving through an ideal two-dimensional material, where the crack tip is a singular point. In real three-dimensional materials, however, tensile cracks are planar objects whose tip forms a propagating one-dimensional singular front. Let us now consider a crack front propagating through a heterogeneous medium populated by an ensemble of localized inhomogeneities (asperities). The front is distorted by its interaction with each asperity. Can the crack front, after many such interactions, still be considered a single coherent entity, or, must the dynamics of failure be described by ensemble of individual cracks in all but the most homogeneous materials? Here we present laboratory measurements of a new type of wave, crack front waves, CFW, which are generated by asperities and propagate along crack fronts in tensile fracture. We will show that CFW are highly localized nonlinear entities that propagate along the front at approximately the Rayleigh wave speed, relative to the material. They possess a characteristic, inherently nonlinear shape, reminiscent of solitons. In glass, whose fracture energy is nearly independent of crack velocity, CFW are very long-lived whereas in PMMA, where the fracture energy increases with crack velocity, CFW decay. CFW serve to both transport and distribute the energy fluctuations, induced by asperities, throughout the entire front. In this way, these waves may allow a crack front to retain its coherence despite repeated interactions with randomly dispersed material inhomogeneities.
\end{abstract}

\section{KEYWORDS Brittle fracture, localized waves, crack front waves, solitary waves}

Dynamic crack propagation in brittle fracture has been the subject of much recent attention. Much of this work has been invested in studying the dynamics and stability of rapidly moving cracks in Mode I fracture. Experiments have shown that above a critical propagation velocity, $v_{c}$, of approximately $0.4 V_{R}$, where $V_{R}$ is the Rayleigh wave speed, a single crack becomes intrinsically unstable. At this speed, a single crack has been observed in brittle [1]becomes unstable to frustrated microscopic branching events. This instability . Qualitatively similar effects have also [11], and recently in crystalline materials[9, 10], glass [1-8]polymers , and molecular [16-18], finite element calculations [12-15]been observed in models of ideal crystals have also demonstrated that the equation of motion for a single [10]. Recent experiments [19, 20]dynamics , provides excellent quantitative agreement with [21]crack predicted by continuum elastic theory experiments in ideal quasi-2D amorphous materials both below and above $\mathrm{v}_{\mathrm{c}}$, whenever a single-crack state (single-crack states can occur momentarily when $\mathrm{v}>\mathrm{v}_{\mathrm{c}}$ ) exists.

In the work summarized above only ideal (defect-free) quasi-2D materials were considered. The tip of a crack is idealized as a singular point progressing through an otherwise perfect two-dimensional material. As long as translational invariance exists in the third dimension, the above experiments have demonstrated that the assumption of two-dimensionality is justified. What happens, however, when the translational invariance in the "ignored" dimension is broken? Let us consider a crack progressing through an, initially, 
ideal three-dimensional plate of finite thickness. Instead of a singular point, the "tip" of a crack is now a singular front, which extends throughout the sample's thickness. Let us now assume that the crack front meets an asperity, i.e. a localized inhomogeneity where the fracture energy is locally either higher or lower than in the surrounding medium. The asperity breaks the system's translational invariance in the direction normal to the crack's motion. We now consider its effect on the crack's motion.

have recently shown, analytically, that the crack's interaction with an asperity [22]Ramanathan and Fisher will induce a new type of wave that will propagate along the crack front. Their analysis was based on Willis calculation of the change in the energy release rate, G, induced by a localized [23] and Movchan's perturbation to a crack front in the propagation direction. This analysis indicated that an asperity could excite a wave, i.e. a local perturbation of the crack's velocity that could progress along the crack front at slightly less than $v_{R}$, relative to the asperity. This disturbance, predicted to exist within the fracture plane, was shown to be marginally stable for constant values of the fracture energy, $\Gamma$. The wave was predicted to [24, grow (decay) if $\Gamma$ were a decreasing (increasing) function of the crack velocity, $v$. Morrissey and Rice have observed these waves in finite element calculations of tensile fracture in elastic 3D materials with a 25] constant fracture energy. They found that asperities along the crack path indeed generated persistent, localized waves of in-plane velocity fluctuations that propagated at velocities slightly below $\mathrm{v}_{\mathrm{R}}$. After an initial decay, these waves continued to propagate along the crack front with constant shape and amplitude.

Below we will describe experiments in which waves, similar in many respects to those predicted above, . In the tensile fracture of soda-lime glass and PMMA, we will show that the interaction [26]were observed of asperities with moving cracks indeed generates localized waves that propagate along the crack front at approximately $\mathrm{v}_{\mathrm{R}}$. The waves are stable in glass (nearly constant $\Gamma$ ) and decay in PMMA ( $\Gamma$ increasing with v). In contrast to the predicted waves, the crack front waves (CFW) observed in experiments have two surprising characteristics: these waves exist both within and normal to the fracture plane and CFW have a unique characteristic profile.

Our experiments were conducted in both PMMA and soda-lime glass plates of size $380 \times 440 \mathrm{~mm}$ and thickness, $h$, between $2<h<6 \mathrm{~mm}$. The cracks were driven in Mode I by applying static tension at the sample's vertical boundaries. We define the $\mathrm{x}$ direction as the direction of propagation of the crack front, $\mathrm{y}$ as the direction of applied tensile stress $(0<\mathrm{y}<440 \mathrm{~mm})$, and $\mathrm{z}$ as direction along the crack front. The plates were initially defect-free. Asperities were generally introduced along the outer faces of the plate $(\mathrm{z}=0$ or $\mathrm{z}=\mathrm{h})$. Asperities with fracture energy less than that of the material were formed by scribing a thin line in the $y$ direction on the plate faces. Asperities with fracture energy greater than that of the material were formed by adhering thin glue lines on the plate faces along the y direction. Both types of disturbances generated $\mathrm{CFW}$ upon interaction with a moving crack. Instantaneous crack velocities were measured at the plate faces

Our velocity resolution at each point along the plate faces was [7]by the technique described in approximately $10 \mathrm{~m} / \mathrm{s}$ in PMMA and $50 \mathrm{~m} / \mathrm{s}$ in glass. Velocity measurements were performed at a $10 \mathrm{MHz}$ sampling rate enabling velocity measurements approximately every $0.2(0.05) \mathrm{mm}$ in glass (PMMA). The velocity measurements were later correlated with fracture surface measurements and optical photographs. The fracture surface profile was mapped to $10 \mathrm{~nm}$ resolution in the y direction by the use of a modified Taylor-Hobson (Surtonic 3+) scanning profilometer with an X-Z spatial resolution of $0.5 \mu \mathrm{m}$. The features on the fracture surface left behind by the CFW were also photographed by the use of incoherent illumination directed through the transparent samples. This light, passing through the fracture surface, was either focused or de-focused as it traversed through any surface features. This effect, similar in character to shadowgraph visualization, enabled the visualization of minute deviations from flatness of the fracture surface.

CFW have both an in-plane and out-of-plane character, in contrast to the in-plane deviations of the front . Evidence of the latter is in the residual tracks (normal to the fracture plane) [22, 24]velocity predicted by that CFW leave behind on the fracture surface. Examples of typical CFW tracks on the fracture surface are shown in Fig. 1. As Fig 1a shows, the tracks are deviations of the fracture surface height. These deviations can be either upward (away from the mean plane of the fracture surface) or downward (into the mean plane of the fracture surface). The direction of these height deviations has no significance, as tracks formed on the both fracture surfaces are mirror images of each other. 

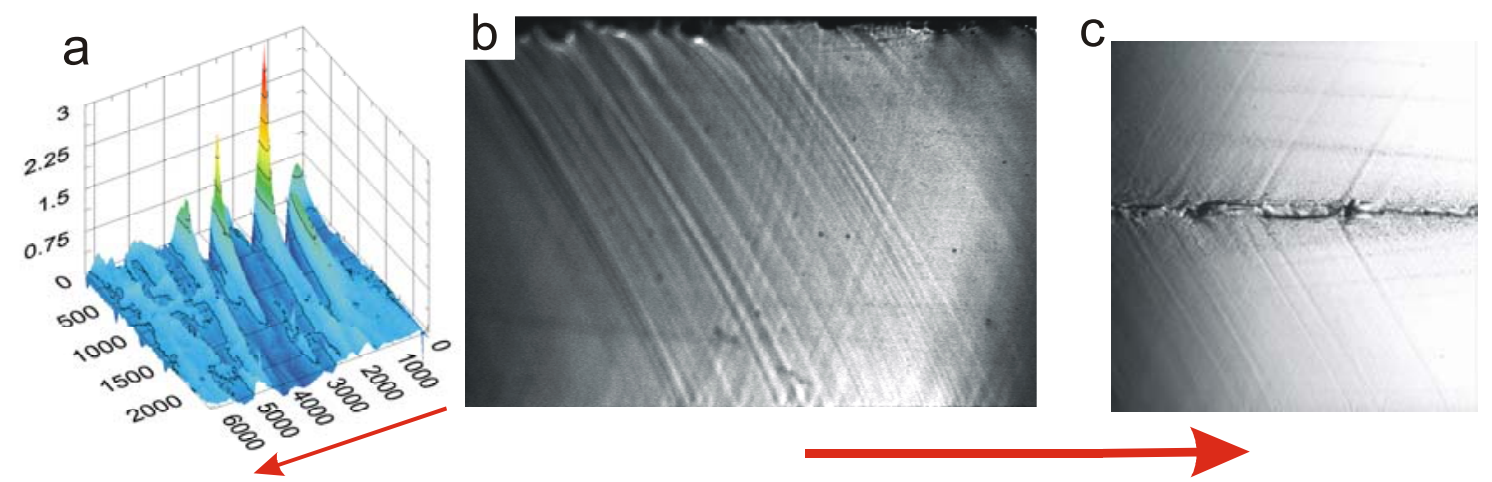

Figure 1. Typical crack front waves generated on a fracture surface. (a) Profilometer measurements (scales are in $\mu \mathrm{m}$ ). The arrow shows the propagation direction. (b) Photograph of front waves generated, as in (a), by an external perturbation at the plate surface. Note both the initial decay (in (a)) and subsequent long lifetime of the waves as they are reflected at the plate surfaces. (c) Photograph of front waves generated by localized micro-branching events. The arrow below (b) and (c) is $3 \mathrm{~mm}$ in length and denotes the propagation

direction.

As the Fig. 1 shows, CFW are generated either by externally imposed asperities (Fig. 1a,b) on the plate surfaces or, intrinsically, by means of micro-branching events. In glass, micro-branching events are generally localized in the $\mathrm{z}$ direction and (as shown in Fig. 1c) occur along lines in the propagation direction . We can understand the initiation of CFW by micro-branching events since, as predicted by theory, the [27] origin of these waves is determined by local fluctuations in the fracture energy. When a micro-branching event occurs, energy is diverted into the daughter cracks that are bifurcating away from the main crack. Thus, from the perspective of the main crack, which was initially the sole source for dissipation in the system, a micro-branching event effectively increases the local value of the fracture energy in the system.
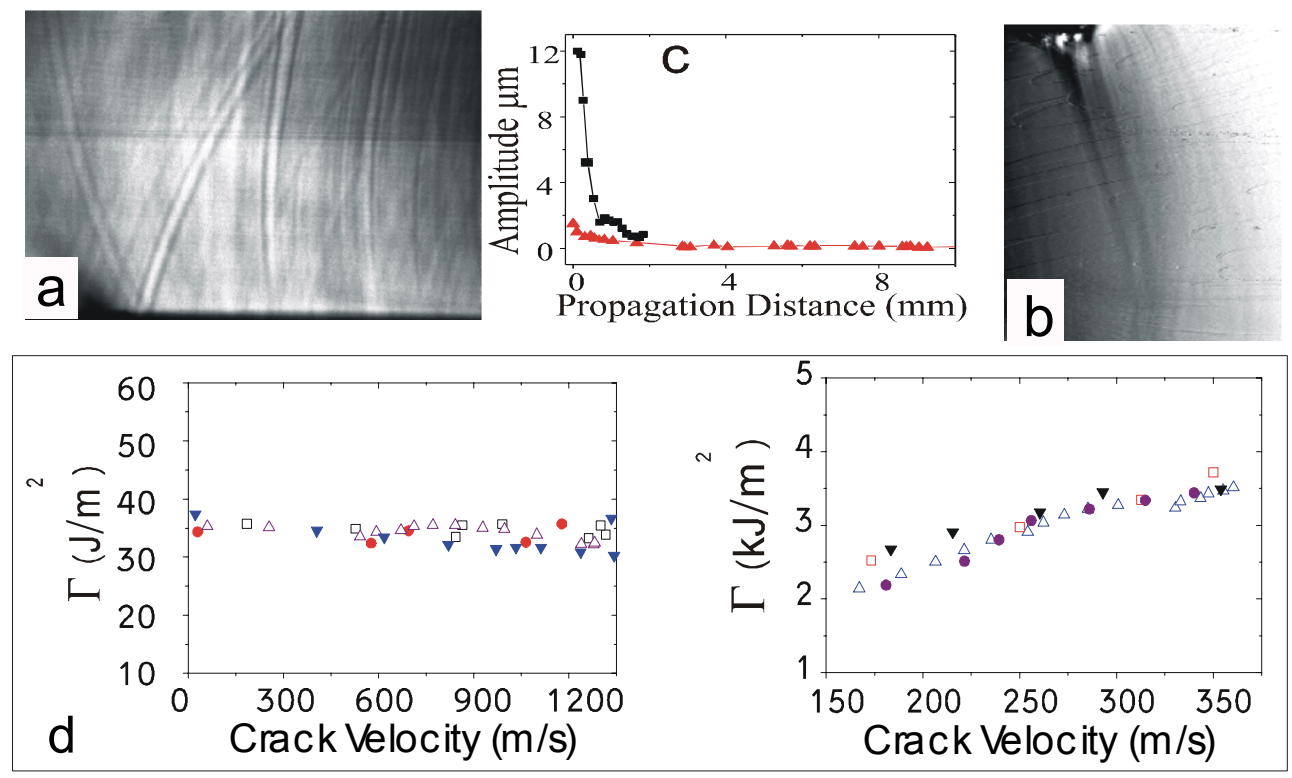

Figure 2. Photographs depicting the evolution of CFW generated by imposed asperities in soda-lime glass (a) and PMMA (b). Both cracks propagate from left to right and each photograph is $3 \mathrm{~mm}$ in height. The amplitude of the waves as a function of their propagation distance is shown in (c). CFW stably propagate in glass whereas in PMMA no stable propagation is observed. (d) The relative stability of CFW in these materials may be explained by the velocity dependence of the fracture energy, $\Gamma$, with $v$ in (right) PMMA and (left) glass.

CFW are not limited to soda-lime glass. In PMMA, as shown in Fig. 2, CFW can also be generated by externally imposed asperities. In PMMA, however, the resulting waves are very quickly damped and have not been observed to stably propagate. In glass, CFW initially decay exponentially with a decay length 
). The waves then stabilize with a constant amplitude [26]scaling with the size of the initial disturbance (see and are, subsequently, very long-lived (Fig. 2c). When impinging on the free [26] and unique shape boundary at a plate's surface, the waves reflect with very little loss of amplitude. We have observed CFW to undergo up to 7 reflections while traveling distances over an order of magnitude greater than their size.

had predicted that CFW are marginally stable if $\Gamma$ is [22]As mentioned earlier, Ramanathan and Fisher independent of $\mathrm{v}$. If $\Gamma(\mathrm{v})$, on the other hand, is an increasing function of $\mathrm{v}-\mathrm{CFW}$ are predicted to decay. The fracture energy dependences of glass and PMMA on $v$ (see Fig. 2d) are consistent with this prediction. While $\Gamma(\mathrm{v})$ for PMMA is a clearly increasing function, $\Gamma$ is nearly constant for glass. The data shown were for velocities prior to the onset of the micro-branching instability. In PMMA we have found [10]obtained that the "bare" $\Gamma(\mathrm{v})$ dependence after the instability. (Effectively, $\Gamma(\mathrm{v})$ will increase for $\mathrm{v}>\mathrm{v}_{\mathrm{c}}$ due to $[8,10]$ the increase in the total surface area formed by both the main crack and micro-branches.)



Figure 3. The front wave velocity can be determined via intersecting CFW tracks for (a) a case where the crack front is normal to the propagation direction and (b) where the front is inclined at an angle $\beta$ relative to the propagation direction. Once $\mathrm{V}_{\mathrm{FW}}=(1 \pm 0.05) \mathrm{V}_{\mathrm{R}}$ is known, this method can be used to measure both the crack velocity, $\mathrm{v}$, and the angle $\beta$ at the point of intersection. (c) Two comparisons of the independent measurements (lines) of $v$ with measurements (points) obtained by means of $v=V_{F W} \cos (\alpha / 2) / \cos (\beta)$. The sharp peaks in $\mathrm{v}$ prior to $\mathrm{v}_{\mathrm{c}}=1500 \mathrm{~m} / \mathrm{s}$ (in glass) result from the crack's interaction with the arrival of asperity-induced front waves.

The velocity of $\mathrm{CFW}, \mathrm{V}_{\mathrm{FW}}$, is very close to the Rayleigh wave speed, $\mathrm{V}_{\mathrm{R}}$, relative to the material. This is . We measure the CFW velocity by means of intersecting CFW tracks [24, 25] and [22]consistent with both on the fracture surface. The intersecting tracks are formed by two distinct counter-propagating CFW. As, in general, CFW can be formed whenever the crack front interacts with any localized material inhomogeneity, intersecting CFW are common. Two such intersections are illustrated in Fig. 3 for cases where the crack front is oriented normal to the propagation direction (Fig. 3a) and at an angle $\beta$ relative to the propagation direction (Fig. 3b). Defining the angle $\alpha$ as the angle formed between two outgoing CFW tracks, $V_{\mathrm{FW}}$ is determined by:

$$
\mathrm{V}_{\mathrm{FW}}=\mathrm{v} \cos (\beta) / \cos (\alpha / 2)
$$


that $\mathrm{V}_{\mathrm{FW}}=\mathrm{V}_{\mathrm{R}}(1 \pm 0.05)$. Once we have established the value of $\mathrm{V}_{\mathrm{FW}}$, we can invert [26]In this way we find Eq. 1 and use the values of $\mathrm{V}_{\mathrm{FW}}, \alpha$ and $\beta$ to measure the instantaneous value of $\mathrm{v}$ at any point where two $\mathrm{CFW}$ tracks intersect. This is demonstrated in Fig. 3c, where independent velocity measurements are in excellent agreement with values of $\mathrm{v}$ obtained by inverting Eq. 1. In many applications where direct measurements of $\mathrm{v}$ cannot be performed, this new tool should prove to be useful.

in the fracture [28]The existence of the tracks left by CFW on fracture surfaces has been noted for decades literature, and identified as "Wallner lines". In most instances, however, their origin has been misinterpreted. Wallner lines are defined as lines imprinted on the fracture surface as a result of the interaction between a moving crack front and shear waves, generated by an external source. The markings on the fracture surface come about as a result of the momentary deflection of the stress field at a crack's tip generated by a passing shear wave. In the well-known Kerchof method, this interaction has been used to advantage as a tool for crack velocity measurement (using the patterns imprinted on the fracture surface by means of externally broadcast, ultrasonic shear waves). CFW however, are not Wallner lines. As illustrated by Fig. 4a, if the fracture surface markings were created by a radially propagating shear wave (generated by a point-like asperity), the tracks would have the following properties. Their amplitude would decrease as $1 / \mathrm{r}^{2}$, their propagation velocity would be that of shear waves, and they would not have a well-defined shape but, instead, mimic the initial conditions that created them. As we have shown (see Fig. 3), the propagation velocity of CFW is approximately $\mathrm{V}_{\mathrm{R}}$, which is more than $2 \sigma$ less than the shear wave velocity in glass. We , CFW amplitudes stabilize [26] have also demonstrated (see Fig. 2) that after an initial exponential decay and these waves continue to propagate large distances with no appreciable change in amplitude. Last, as of the initial [26]shown in Fig. 4b, CFW have a unique, well-defined profile whose shape is independent conditions that formed them. Thus, although CFW are superficially similar to Wallner lines, the aforementioned properties show them to be qualitatively different entities.
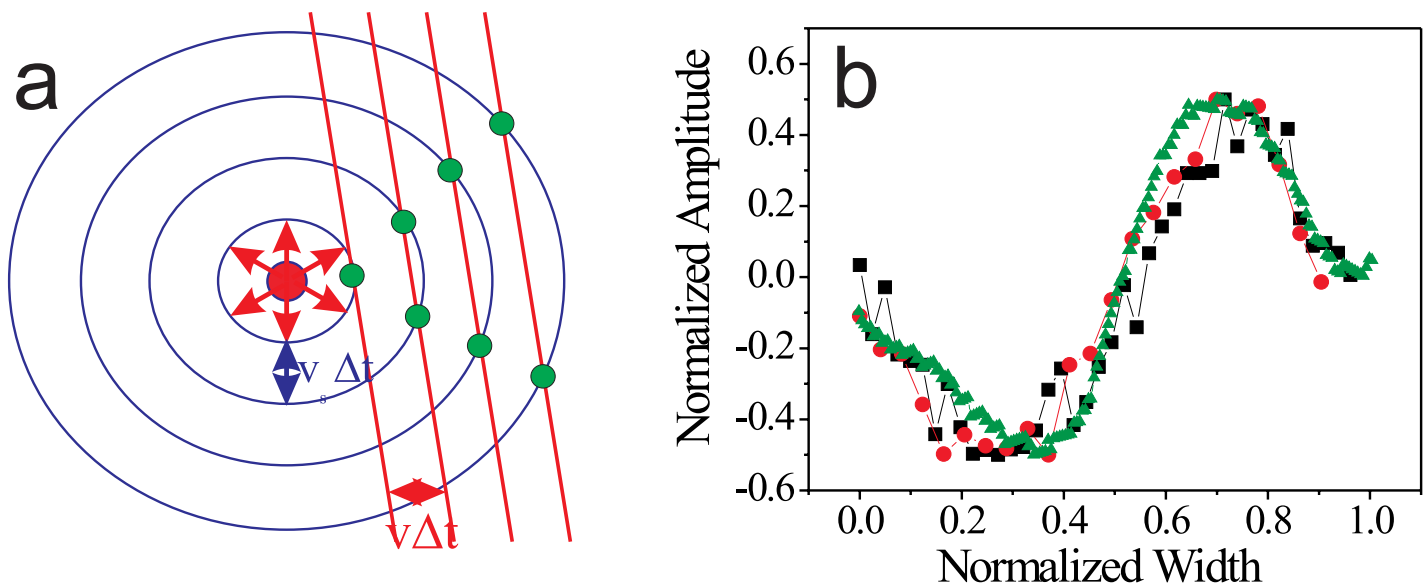

Figure 4. (a) A schematic picture depicting how surface markings might be formed by the interaction between the crack front and shear waves (i.e. the "Wallner line" mechanism). CFW properties are not . Shown are [26]consistent (see text) with this scenario. (b) CFW have a unique characteristic profile superimposed profiles of 3 different front waves. Each profile is scaled by the size of the initial asperity that formed it. The initial forms of the profiles used in (c) were very much different from their asymptotic profiles. The scales of the three profiles shown span over an order of magnitude.

In conclusion, crack front waves appear to be a new type of elastic wave. Although they "live" on a crack front, they move at a constant velocity of approximately the Rayleigh wave speed relative to the medium. CFW are not linear waves. Their characteristic shape provides evidence of a nonlinear, soliton-like character. Upon interaction CFW are not destroyed but retain both their shape and amplitude [26]. In addition, these highly localized waves transfer energy [27] throughout the fracture surface. This together with their propagating nature may enable them to allow a crack to remain a single coherent entity - even in highly inhomogeneous materials, since, statistically, any local changes to the crack front induced by a given asperity will, by means of CFW, be distributed throughout the entire front.

ACKNOWLEDGEMENTS: We wish to acknowledge the support of the US-Israel Binational Fund. 


\section{REFERENCES}

1. Fineberg, J. and M. Marder, (1999) Physics Reports 313(1-2) p. 2.

2. $\quad$ Fineberg, J., et al., (1991) Physical Review Letters 67(4) p. 457.

3. Fineberg, J., et al., (1992) Physical Review B-Condensed Matter 45(10) p. 5146.

4. Hauch, J.A., et al., (1999) Physical Review Letters 82(19) p. 3823.

5. Boudet, J.F., S. Ciliberto, and V. Steinberg, (1996) Journal De Physique II 6(10) p. 1493.

6. Sharon, E., S.P. Gross, and J. Fineberg, (1995) Physical Review Letters 74(25) p. 5096.

7. Sharon, E. and J. Fineberg, (1996) Physical Review B54(10) p. 7128.

8. Sharon, E., S.P. Gross, and J. Fineberg, (1996) Physical Review Letters 76(12) p. 2117.

9. Sharon, E. and J. Fineberg, (1998) Philosophical Magazine B78(2) p. 243.

10. Sharon, E. and J. Fineberg, (1999) Nature 397(6717) p. 333.

11. Cramer, T., A. Wanner, and P. Gumbsch, (2000) Physical Review Letters 85 p. 788.

12. Marder, M. and X.M. Liu, (1993) Physical Review Letters 71(15) p. 2417.

13. Marder, M. and S. Gross, (1995) Journal of the Mechanics and Physics of Solids 43(1) p. 1.

14. Kessler, D. and H. Levine, (2001) Physical Review E59 p. 5154.

15. Astrom, J. and J. Timomen, (1996) Physical Review B54 p. 9585.

16. Miller, O., L.B. Freund, and A. Needleman, (1999) Modelling and Simulation in Materials Science and Engineering 7(4) p. 573.

17. Xu, X.P. and A. Needleman, (1994) Journal of the Mechanics and Physics of Solids 42(9) p. 1397.

18. Johnson, E., (1992) International Journal of Fracture 57(2) p. R27.

19. Holland, D. and M. Marder, (1998) Physical Review Letters 80(4) p. 746.

20. Abraham, F.F., et al., (1994) Physical Review Letters 73(2) p. 272.

21. Freund, L.B., Dynamic Fracture Mechanics. 1990, New York: Cambridge.

22. Ramanathan, S. and D.S. Fisher, (1997) Physical Review Letters 79(5) p. 877.

23. Willis, J.R. and A.B. Movchan, (1995) Journal of the Mechanics and Physics of Solids 43(3) p. 319.

24. Morrissey, J.W. and J.R. Rice, (1998) Journal of the Mechanics and Physics of Solids 46(3) p. 467.

25. Morrissey, J.W. and J.R. Rice, (2000) Journal of the Mechanics and Physics of Solids 48(6-7) p. 1229.

26. Sharon, E., G. Cohen, and J. Fineberg, (2001) Nature (to be published).

27. Sharon, E., G. Cohen, and J. Fineberg, (2001) (in preparation)

28. Wallner, H., (1939) Z. Physik 114 p. 368. 